\title{
The Revolution of Artificial Intelligence and the Significance of Adopting AI in different Industries
}

\author{
Shanmugasundaram Palanimalai
}

\begin{abstract}
Artificial Intelligence (AI) is one of the most widely inflated technologies in several industries today. With the emergence of IoT, Big data and Digitalization, many industries produce large sets of data and AI begins to be the prominence for solving the increasing number of complications in this relevance. Artificial Intelligence (AI) and Machine Learning (ML) applications, spectacle substantial guarantee in gaining commercial traction in several businesses as AI brings with a probable of genuine human-to-machine interaction. When machines become intelligent, they can understand needs, connect with data points and arrive at better decisions. Therefore, Artificial Intelligence (AI) and Machine Learning technologies are being quickly adopted in wide range of applications in several industries. In this paper, we epitomize the fundamentals and the significance of adopting of Artificial Intelligence technologies in different industries.
\end{abstract}

Keywords: Artificial Intelligence, Machine Learning, Data Analytics.

\section{INTRODUCTION}

According to John McCarthy (One of the founders of AI), $\mathrm{AI}$ is "the science and engineering of making intelligent machines, especially intelligent computer programs". In other words, AI is a way of making a computer, a computer controlled robot (or) software that think intelligently like how humans would think. The aim of AI is to produce machines with computer programs that have intellectual capabilities to analyze their environment (or) situation and make decisions like a human. AI is a vast field and there are many areas within it but eventually it is about making decisions cognitively. Machine learning is the foundation (or) building block of AI. Machine Learning predominantly is concerned with making the right decision based on learning. Deep Learning is a subset of machine learning. Deep Learning is predicative and it understands accuracy, gives metrics or guidelines to learn on its own without supervision from humans.

Artificial Intelligence technologies have led to several breakthroughs in the field. We highlight the some of the major millstones of AI developments in the below figure-1.

Revised Manuscript Received on December 05, 2019

* Correspondence Author

Dr.Shanmugasundaram Palanimalai *, Project Manager - Information Technology, Bangalore, India.

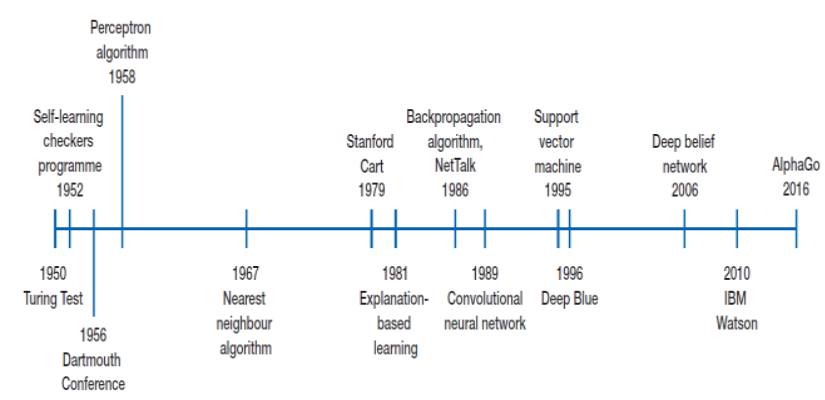

Fig. 1. Major Millstones of AI developments (Source: IEC- White Paper on AI across industries)

$\mathrm{AI}$ is a wide topic and has several subsets. In the below we illustrate the distinction between Artificial Intelligence, Machine learning and Deep learning.

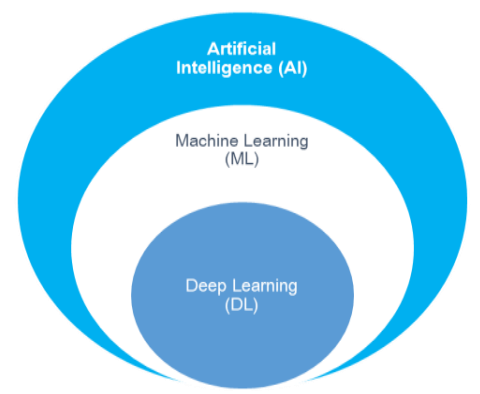

Fig. 2. Distinction between AI, ML \& Deep Learning

\section{A. Artificial Intelligence}

In a broader sense, Artificial Intelligence (AI) is a science and a set of computational technologies that brings ability to machines and computer systems to think and work like a human. It further adds talents like speech recognition, visual perception, decision-making, emotion comprehension and more into systems.

\section{B. Machine Learning}

ML is a subset of AI that allows machines to automatically learn and improve from experience. Specialized systems are made for this purpose and no explicit programming is required to add new definitions to the database. The machines can learn on their own. It is nothing but the advancement of computer algorithms and applications, which will allow the systems to 
access massive amount of data and learn from them to complement particular ideas (or) tasks.

\section{Deep Learning}

Deep learning is a subset of machine learning that consists of particularly large neural networks and a massive collection of algorithms that can imitate human intelligence. DL will provide the ability to learn from data sets that are unlabeled or unstructured. One of the complications that make deep learning stand out is the fact that the networks in this technology are unsupervised, meaning they are continuously learning without human intervention.

\section{PROMISING APPLICATION FOR ARTIFICIAL INTELLIGENCE}

With the mounting advancement of AI, many enterprises observe the value in adopting AI and Machine learning to address their business challenges. As industries, want faster and easy way to bring AI to their organizations to draw a successful promising human-to-machine interaction and arrive at enhanced conclusions. The below picture illustrates the promising application for AI.

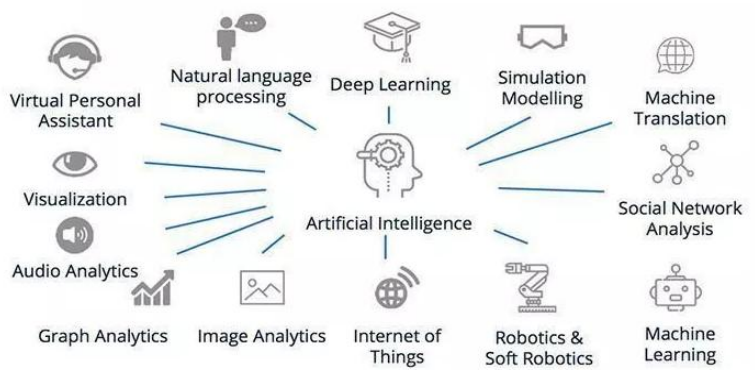

Fig. 3. Promising Application for Artificial Intelligence

\section{THE ROLE OF DATA IN ARTIFICIAL INTELLIGENCE \& MACHINE LEARNING WORKFLOW}

In the digital transformation era, Artificial intelligence (AI) \& Machine learning (ML) are the incipient technologies that will enable the enterprises, uncovered insights sealed in the enormous volume of data, being generated on a daily basis. The AI and ML technologies enable the organization to discover the insights through intensive data analytics for making better and faster decisions. As per ZK Research forecast, by 2025 , there will be 80 billion connected endpoints; each of them will generate significant volumes of data. Therefore, the development of AI and ML technologies will grow considerably as this will aid the organization to discover the insights through intensive data analytics for making better and faster.

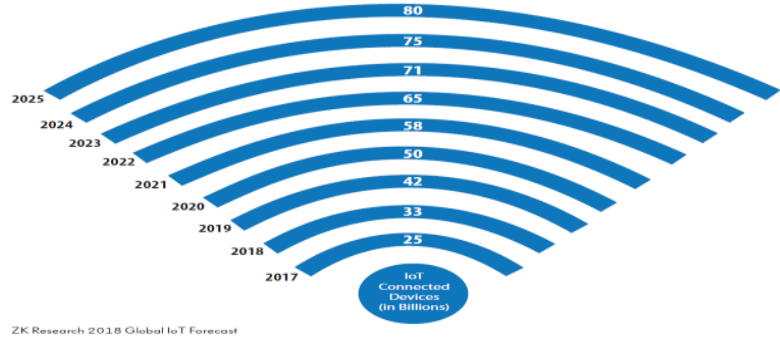

Fig. 4. IoT connected Devices in Billions

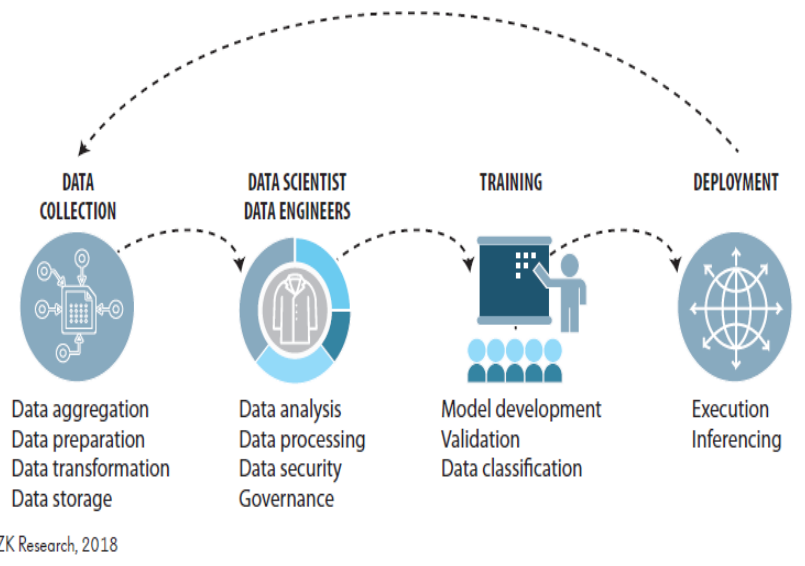

Fig. 5. The Role of Data in AI/MI Workflow

\section{THE SIGNIFICANCE ADOPTING AI TECHNOLOGIES IN DIFFERENT INDUSTRIES}

In the below we explain the importance of having AI technologies in different industries.

\section{A. Automation and learning through data}

AI Automates the monotonous learning and detection through data i.e. AI executes frequent, higher volume and computerized tasks consistently without any fatigue.

\section{B. Bringing higher intellectual to enhance existing applications}

AI brings improved intellectual to existing products. In many circumstances. AI will not be given as an individual application. Rather, products that are already in use, will be further improved with AI capabilities. Automation, conversational platforms and smart machines can be combined with huge amounts of data to improve various technologies in the workplace.

\section{AI acclimates through progressive learning algorithms}

AI acclimates through progressive learning algorithms to let the data do the programming. AI discovers structure and regularities in data so that the algorithm obtains a skill and the algorithm turn out to be a classifier (or) a predictor. Therefore, just as the algorithm can teach itself how to do the action by learning through the data. An AI technique, that lets the model to adjust, through training and added data when the first response is not right.

\section{AI performs deeper data Analysis}

AI analyzes more and deeper data by using neural networks, which have various hidden layers. Making a fraud detection system with five hidden layers was practically very difficult a few years ago, however all that has changed with incredible evaluation of big data. We want huge data to train deep learning models since they learn directly from the data. The more data we can feed them, the more accurate they become.

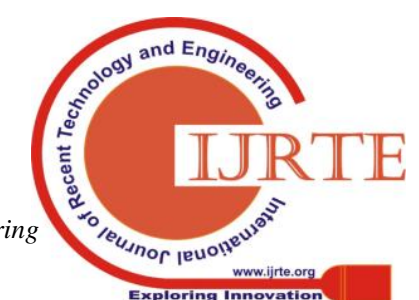




\section{E. Incredible accuracy acquires most out of data}

AI accomplishes unbelievable accuracy through deep neural networks that was previously very difficult to achieve. For instance, interactions with Google Search \& Google Photos are all based on deep learning and they keep getting more accurate the more we use them. Furthermore, AI acquires the most out of data. While algorithms are self-learning, the data itself can become intelligent property. The answers are in the data; we just have to apply AI to get them out. Meanwhile the role of the data is now more imperative than ever before, it can make a viable advantage.

\section{CONCLUSION}

In this paper, we have illustrated the fundamentals of Artificial Intelligence and the significance of adopting AI in different industries. AI is one of the chief components for new safety concepts, more particularly when it comes to handling machines. As AI is already having an insightful, impact on society, industries and people, it is now expected to become one of the key driving forces for fundamental innovation in many industry sectors i.e. manufacturing, Retail, Finance, whether, energy, education, transportation or healthcare.

\section{REFERENCES}

1. TURING, A. M., Computing Machinery and Intelligence, Mind 49, 1950, pp. 433-460.

2. MCCARTHY, J., MINSKY, M. L., ROCHESTER, N., SHANNON, C. E., A Proposal for the Dartmouth Summer Research Project on Artificial Intelligence, Original from August 31, 1955, reprint in AI Magazine, Vol. 27 (4), 2006.

3. Computer Research Association 2012. Challenges and Opportunities with Big Data - A community white paper developed by leading researchers across the USA.

4. IDC Spending Guide Forecasts Worldwide Spending on Cognitive and Artificial Intelligence Systems to Reach \$57.6 Billion in 2021 [Online]. Available: go.iec.ch/wpai001. [Accessed: 14 September 2018].

5. WOLFE, F., How Artificial Intelligence Will Revolutionize the Energy Industry [Online]. Available: go.iec.ch/wpai005. [Accessed: 14 September 2018].

6. PwC, Demographic and social change [Online]. Available: go.iec.ch/wpai006. [Accessed: 14 September 2018].

7. International Telecommunication Union, AI for Good Global Summit Report [Online]. Available: go.iec.ch/wpai007. [Accessed: 14 September 2018].

8. CHAKRABORTY, C. and JOSEPH, A., Bank of England: Staff Working Paper No.674, Machinelearning at central banks [Online]. Available: go.iec.ch/wpai009. [Accessed: 14 September 2018].

9. IEC, Edge intelligence, White Paper, 2017 [Online]. Available: go.iec.ch/wpai002. [Accessed: 14 September 2018].

10. Statista, Adoption level of AI in business organizations worldwide, as of 2017 [Online]. Available:go.iec.ch/wpai010. [Accessed: 14 September 2018].

11. McKinsey Global Institute, Artificial Intelligence: implications for China [Online]. Available: go.iec.ch/wpai011. [Accessed: 14 September 2018].

12. SAP, European Prosperity Through Human-Centric Artificial Intelligence [Online]. Available: go.iec.ch/wpai012. [Accessed: 14 September 2018]. 